\title{
A Novel Segmentation and Contouring Scheme to Assist Accurate Brain Lesion Classification
}

\author{
${ }^{1}$ Madhukumar, S. and ${ }^{2}$ N.Santhiyakumari \\ ${ }^{1}$ Department of Electronics and Communication Engineering, \\ St Joseph's College of Engineering and Technology, Palai, Kottayam, India \\ ${ }^{2}$ Department of Electronics and Communication Engineering, \\ Knowledge Institute of Technology, Salem, India \\ ${ }^{1}$ madlekarthi@gmail.com, 2santhiyarajee@rediffmail.com
}

\begin{abstract}
Segmentation of highly infiltrating glioblastoma multiforme (GBM) in BrainMR Images has been highly challenging as the grey levels of tumor and peritumoral vasogenic edema are quite homogenous and hence identifying a suitable scheme for isolating GBM from the background has been troublesome.This paper proposes a novel segmentation and contouring scheme, using shape sensitive derivative strategy for segmentation and energy minimizing contours for enhancing the edges of the GBM, under investigation. The efficiency of the algorithm has been tested with the aid of extracted tumor features, the Shape Features -circularity, irregularity, Area, Perimeter, Shape Index, Intensity features - Mean, Variance, Standard Variance, Median Intensity, Skewness, and Kurtosis, Texture features -Contrast, Correlation, Entropy, Energy, Homogeneity, cluster shade, sum of square variance. It is obvious, though this algorithm consumes more computational time, it segments the edges effectively and preserve the shape facilitating accurate extraction or estimation of the features further and provides stable and reproducible results. All the classification Schemes, with combined DA and SVM with Higher Rank Features and LDA techniques exhibited appreciable improvement in terms of sensitivity, specificity, positive Predictive value and negative Predictive value, because of the accuracy of shape sensitive derivative segmentation algorithm and energy minimizing contour algorithm.
\end{abstract}

Keywords: Magnetic Resonance Brain images, class separable shape sensitive segmentation, Energy Minimizing Contours, Image Enhancement.

\section{Introduction}

Segmentation of Brain structures, for estimation of various parameters, is one of the primary steps, towards the design of efficient image based Clinical Decision Support Systems (CDSS). The main aim of segmentation in CDSS is to recognise homogeneous regions within an image as distinct features and enable them to be classified as different objects. [1] The segmentation process can be based on finding the maximum homogeneity in grey levels within the regions identified. One of the common problems encountered in image segmentation is choosing a suitable approach for isolating different objects from the background. [2] The segmentation does not perform well if the grey levels of different objects are 
quite similar as in the case of B mode Magnetic Resonance. The presence of speckling artefacts further increases the complexity of the system. It is expected that an ideal segmentation scheme will enhance the boundary differences between the objects and their background making the parameter estimation task easier and accurate. [3] Other issues related to segmentation involve choosing good segmentation algorithm, measuring their performance, and understanding their impact on the scene analysis system. Classical image segmentation techniques are based on two pixel characteristics: discontinuities and similarities. Discontinuity is an approach to partition the image based on abrupt changes in intensity (or) gray level. Similarity is an approach based on partitioning an image into regions according to a set of pre-defined criteria. [4] Many of the classical techniques like Region growing, a procedure that groups pixels (or) sub regions into larger regions have been applied to try to solve this problem with variable outcomes. Such techniques tend to be unreliable when segmenting a structure that is surrounded by others with similar image intensity (e.g., low-contrast structures). More sophisticated techniques, like level set method (LSM) is a numerical technique for tracking interfaces and shapes. The advantage of the level set method is that one can perform numerical computations involving curves and surfaces on a fixed Cartesian grid without having to parameterize these objects), use more powerful computations for tracking the evolution of moving surface. [5] An initial approximation of the solution evolves until it gets the limits of the region of interest. In this paper, the shape sensitive derivative approach which quantifies the sensitivity of the problem when the domain under consideration is perturbed by changing the material property at a specific point has been utilized. The problem of segmentation of Magnetic Resonance images has been achieved via optimization of a cost function by asymptotic expansion. Optimal shape estimation plays a vital role in feature estimation and pattern recognition techniques. The segmented images are contoured using energy minimizing contours. This novel scheme has been tested on Brain images for estimating the features for providing enhanced Obstetric care. Experiments were carried out with first, second and third trimester Brain images and found to produce stable and reproducible results.

\section{Derivative Based Image Segmentation Algorithm}

Feature segmentation of Magnetic Resonance images is a multi-disciplinary challenge including expertise in signal and image processing, optimization theory and requires a detailed anatomical knowledge of the subject under study. Wide variety of segmentation schemes can be found in the literature and these can be broadly categorized as Boundary-based, Region based, Shape Model approaches and thresholding schemes. Boundary based algorithms extract the region of interest based upon the intensity variation at the edges as they expect the ROI to have some perfect demarcation at the edges. These algorithms can further be grouped into optimum boundary based schemes; active contours based algorithms and level sets [6]. Another group of segmentation schemes are the region based methods which extract the region of interest based on the similarity measure. A seed pixel is initialized first and the predefined similarity measure is used to evaluate the degree of similarity between the pixels. A number of region based schemes are present in the literatures which are based on clustering, graph cut, fuzzy connectedness, MRF, watersheds and optimum partitioning (Mumford-Shah, Chan-Vese). Another classification of the segmentation algorithms are the Shape Model approaches [2] [7]. These algorithms play a vital role in real time applications are focused much in the recent years by researchers. They tend to extract out the features based upon the prior knowledge of the shape of the 
Madhukumar.S and N.Santhiyakumari; A Novel Segmentation and Contouring Scheme to Assist Accurate Brain Lesion Classification, Journal of Biomedical Engineering and Medical Imaging, Volume 1, No 6, Dec (2014) , pp 1-9

feature to be extracted. Some of the Shape based approaches cited in the literature are manual tracing methods, Live wire techniques, Active Shape and Active Appearance approaches and atlas-based methods [8] [9] [10].

The introduction of the Topological Derivative allows to quantify the sensitivity of cost function when domain under consideration is perturbed, and it indicates the best place where perturbation could be introduced. The topological shape sensitive derivative is computed for an appropriate functional associated to the image indicating the cost endowed to specific image segmentation [8].

The image segmentation algorithm based on this derivative can be proposed as follows. Let $\mathcal{J}(\Omega)=$ $\mathcal{J}(\phi(\Omega))$ be the cost function to be minimized and $\phi(\Omega)$ the solution of an associated variational problem (VP) defined in the domain $(\Omega)$. For a small parameter $\epsilon \geq 0$, let $\Omega_{\epsilon}$ be the perturbed domain obtained by the insertion of heterogeneity on the parameters governing the associated VP. This heterogeneity has been defined in a small ball of radius $\epsilon$ centred at any point $\hat{x}$ of the domain $\Omega$. Furthermore, let $\phi_{\epsilon}$ be the solution of the VP defined in the perturbed domain $\Omega_{\epsilon}$. Then, for small values of parameter $\epsilon$ the topological sensitivity provides an asymptotic expansion of $\mathcal{J}\left(\Omega_{\epsilon}\right)$ (it is a series of functions which has the property of truncating the series after a finite number of terms provided an approximation to a given function).

$$
\mathcal{J}\left(\Omega_{\epsilon}\right)=\mathcal{J}(\Omega)+f(\epsilon) D_{T}(\hat{x})+o(f(\epsilon))
$$

Where, $\mathcal{J}\left(\Omega_{\epsilon}\right)$ is the shape derivative of cost function in relation to the parameter $\epsilon, f(\epsilon)$ is the positive function going monotonically to zero with $\epsilon$ and $D_{T}(\hat{x})$ is the Topological derivative. This derivative can be seen as a first order correction on $\mathcal{J}(\Omega)$ to estimate $\mathcal{J}\left(\Omega_{\epsilon}\right)$. Since $f(\epsilon)$ is positive, the heterogeneity must be introduced at any point $\hat{x}$ where $\mathrm{D}_{\mathrm{T}}$ is negative in order to reduce the value of the cost function J. The topological derivative can be easily obtained and this segmentation method appears robust even in the presence of very large noise in the image data. Initially we present the formulation of the segmentation problem. In particular, we define the cost functional associated to a specific segmentation of the image data. We also define the variational problem (VP) which characterizes function $\phi$. The proposed segmentation algorithm and numerical approximation used to find an approximated solution of the associated VP [8].

\subsection{Problem Formulation}

The image data is normally characterized by a two-dimensional matrix of pixels or a three dimensional matrix of voxels, which is the brightness level or grey level intensity at that point. Thus, to each image element is associated an intensity. The original image data can be described by a real valued functions $v$ which is constant at image element level, then,

$$
v \in \mathcal{V}=\left\{w \in L^{2}(\Omega)\right\}
$$

Where,

$\mathcal{V}$ - Close subspace of domain.

$w$ - Constant at image element level.

$L$ - Limit point of the domain.

$\Omega$ - An open bounded domain in $\mathbb{R}^{n}, n=2,3$. 
In addition let us define the set as classes $C$,

$$
C=\left\{c_{i} \in \mathbb{R}: \mathrm{i}=1, \cdots \mathrm{N}_{\mathrm{c}}\right\}
$$

Where, $\mathrm{N}_{c}$ is the number of predefined classes in which the original image $U$ will be segmented. $\mathrm{c}_{\mathrm{i}}$-Represents the intensity that characterizes the $i^{\text {th }}$ class. The image segmentation problem can be stated as following: Given the image data $u \in \mathcal{V}$ find the segmented image $u^{*} \in U$ such that minimizes a functional $\mathcal{J}: U \rightarrow \mathbb{R}$ endowed to the cost of a specific segmented image and being $U$ defined as,

$$
U=\{\mathrm{u} \in \mathcal{V}: u(x) \in C \forall x \in \Omega\}
$$

The degree of match between an image and segmentation can be best estimated using Mumford and Shah functional measure. Hence by Mumford and Shah functional, the cost functional $J$ associated to a segmented image $u \in U$ can be given by,

$$
\mathcal{J}(\Omega)=\frac{1}{2} \int_{\Omega} \mathbb{K} \nabla \varphi \cdot \nabla \varphi d \Omega+\frac{1}{2} \int_{\Omega}(\varphi-(\mathrm{v}-u))^{2} d \Omega
$$

Where field $\phi$ is solution of the following variational problem: Find $\phi \in \mathrm{H}^{1}(\Omega)$ such that,

$$
a(\varphi, \eta)=l(\eta) \forall \eta \in \mathrm{H}^{1}(\Omega)
$$

With the Bilinear form $a(\cdot, \cdot)-\mathrm{H}^{1}(\Omega) \times \mathrm{H}^{1}(\Omega) \rightarrow \mathrm{R}$.

Linear form $I(\cdot)-L^{2}(\Omega) \rightarrow R$.

The bilinear and linear form of the variational problem can be redefined as

$$
\begin{gathered}
a(\varphi, \eta)=\int_{\Omega} \mathbb{K} \nabla \varphi \cdot \nabla \eta \mathrm{d} \Omega+\int_{\Omega} \varphi \eta \mathrm{d} \Omega \\
l(\eta)=\beta \int_{\Omega}(\mathrm{v}-\mathrm{u}) \eta \mathrm{d} \Omega
\end{gathered}
$$

Where, $\beta$ is the parameter chosen experimentally and $\mathbb{K}$ is the diffusivity of second order tensor field which is constant at image element level. The existence and uniqueness of the solution $\phi$ of the variational problem can be estimated through Lax-Milgram theorem.

Associated to $\phi$ is defined the function $\phi_{\epsilon}$ solution of a perturbed variational formulation. The perturbation has been characterized by changing the segmented image $u$ with a new one $u_{T}$ which is identical to $u$ at every point of the domain $\Omega$ except in the small region $\mathrm{B}_{\epsilon}$ centered at point $\hat{x} \in \Omega$.

In $\mathrm{B}_{\epsilon}, u_{\mathrm{T}}$ assumes one of the values $c_{i} \in C$. Formally,

$$
\begin{aligned}
& u_{T}(x)=u(x) \forall x \in \Omega \backslash \overline{B \epsilon} \\
& u_{T}(x)=c_{i} . c_{i} \in C \forall x \in B_{\epsilon}
\end{aligned}
$$

Therefore, the perturbed cost functional becomes

$$
\mathcal{J}\left(\Omega_{\epsilon}\right)=1 / 2 \int_{\Omega} \mathbb{K} \nabla \varphi_{\epsilon} \cdot \nabla \varphi_{\epsilon} d \Omega+1 / 2 \int_{\Omega}\left(\varphi_{\epsilon}-\left(v-u_{T}\right)\right)^{2} d \Omega
$$

Where field $\phi_{\epsilon}$ is solution of the perturbed variational problem: Find $\phi_{\epsilon} \in H^{1}(\Omega)$ such that:

$$
a\left(\varphi_{\epsilon}, \eta\right)=l_{\epsilon}(\eta) \quad \forall \eta \in H^{1}(\Omega)
$$


Madhukumar.S and N.Santhiyakumari; A Novel Segmentation and Contouring Scheme to Assist Accurate Brain Lesion Classification, Journal of Biomedical Engineering and Medical Imaging, Volume 1, No 6, Dec (2014) , pp 1-9

With $/(\cdot): L^{2}(\Omega) \rightarrow \mathbb{R}$ defined as:

$$
l_{\epsilon}(\eta)=\beta \int_{\Omega}\left(v-u_{T}\right) \eta d \Omega
$$

Satisfying the same properties established by expressions (9-11). Moreover, from these properties the following estimate holds,

$$
\left\|\varphi_{\epsilon}-\varphi\right\|_{H^{1}(\Omega)} \leq C\left|B_{\epsilon}\right|^{1 / 2}
$$

Where, $C$ is the Constant independent of $\epsilon$ and $\left|B_{\epsilon}\right|$ is the Lebesgue measure of $B_{\epsilon}$.

In mathematics, the Lebesgue measure, named after Henri Lebesgue, is the standard way of assigning a length, area or volume to subsets. It has used throughout real analysis, in particular to define Lebesgue integration. Sets which can be assigned a volume are called Lebesgue measurable, the volume or measure of the Lebesgue measurable set $A$ is denoted by $\lambda(A)$. A Lebesgue measure of $\infty$ is possible, but even so, assuming the axiom of choice, not all subsets of $\mathrm{R}^{n}$ are Lebesgue measurable. Lebesgue measure is often denoted $\mathrm{d} x$, but this should not be confused with the distinct notion of a volume form.

\subsection{Computation of the Const Function}

The topological derivative allows us to quantify the sensitivity of the problem when the domain under consideration is perturbed by introducing a hole, an inclusion or a source term in a small region $B \in\left(B_{\epsilon}\right.$ is a ball of radius $\epsilon)$. The topological derivative is given by the following limit $(\epsilon \rightarrow 0)$, [11]

$$
D_{T}(\hat{x})=\lim _{\epsilon \rightarrow 0} \frac{\mathcal{J}\left(\Omega_{\epsilon}\right)-\mathcal{J}(\Omega)}{f(\epsilon)}
$$

Using the Topological-Shape Sensitivity Method, the topological derivative can be also written as:

$$
D_{T}(\hat{x})=\lim _{\epsilon \rightarrow 0} \frac{1}{f^{\prime}(\epsilon)} \frac{d}{d \epsilon} \mathcal{J}\left(\Omega_{\epsilon}\right)
$$

Where the derivative of the cost function with respect to the parameter $\epsilon$ may be seen as its classical shape derivative. Applying Renold Transport Theorem and simplifying using tensorial equations, the localized cost function for segmentation can be obtained as

$$
D_{T}(\hat{x})=1 / 2\left(c_{i}-u\right)\left[(\varphi(\hat{x})-(v-u))+\left(\varphi(\hat{x})-\left(v-c_{i}\right)\right) 2(1-\beta) \varphi(\hat{x})\right] \forall \hat{x} \in
$$

The topological derivative at any point $\hat{x} \in \Omega$ only depends on the value at that point of the function $\phi$ solution of the variational problem given by (6) defined in the non-perturbed domain $\Omega$, on the image data $v$, on the actual segmented image $u$ and on the perturbation given by one of the intensity values characterizing the $\mathrm{N} c$ classes $c_{i} \in C$ in which the image data $v$ will be segmented. Moreover, from (1) and since $f(\epsilon)$ is positive, by introducing a perturbation at any point $\hat{\mathrm{x}}$ where $\mathrm{D}_{\top}(\hat{\mathrm{x}})$ is negative we will obtain a cutback on the cost function value. Then, $\mathrm{D}_{T}$ can be taken as an indicator function defining the best places where the perturbations could be introduced. Since the solution $\phi$ of the variational problem given by (23) cannot, in general, be known explicitly an approximate solution is mandatory. To this end the Finite Element Method will be adopted for the numerical experiments to be shown later. The finite element method (FEM) is a numerical technique for finding approximate solutions of partial differential equations (PDE) as well as of integral equations [12]. 
The solution approach is based either on eliminating the differential equation completely (steady state problems), or rendering the PDE into an approximating system of ordinary differential equations, which are then numerically integrated using standard techniques such as Euler's method, Runge-Kutta etc. Using the simplest finite element given by linear quadrilateral (for two-dimensional image data) or by linear parallelepiped (for three-dimensional image data) with nodal points coincident with the centres of the image elements an approximate solution $\phi^{\text {h }}$ of $\phi$ will be easily obtained for any image data $v \in \mathcal{V}$ and segmented image $u \in U$. Using this solution, a finite element approximation of the topological derivative takes the form,

$$
D_{T^{h}}(\hat{x})=1 / 2\left(c_{i}-u^{h}\right)\left[\left(\varphi^{h}(\hat{x})-\left(v^{h}-u^{h}\right)\right)+\left(\varphi^{h}(\hat{x})-\left(v^{h}-c_{i}\right)\right)+2(1-\beta) \varphi^{h}(\hat{x})\right] \forall \hat{x} \in \Omega
$$

Where $v^{\mathrm{h}}$ and $u^{\mathrm{h}}$ are the finite element interpolation at point $\hat{\mathrm{x}}$ of the functions $v$ and $u$ respectively. Furthermore, considering that the topological derivative depends on $c_{i}$ let us denote by $\widehat{c l}$ the class $c_{i}$ which minimizes $D_{T}^{h}(\hat{x})$ that we will also denote by $\widehat{D_{T^{h}}(\hat{x})}$. As mentioned before, according to the topological asymptotic expansion in (1), for an image data $v \in \mathcal{V}$ we must find the segmented image $u^{*}$ $\in U$ which minimizes the cost functional $\mathcal{J}$ by successively choosing the class that produces the most negative values of the topological derivative [12].

\subsection{Contouring Scheme}

The contour approaches [13] are widely used in practical exploitation of data from images because they effectively use specific prior information about objects and this makes them inherently efficient algorithms. Furthermore, active contours apply processing algorithms selectively to regions of the image, rather than processing the entire image [8]. In general, the energy function of contour model (snake) [14] can be given by

$$
E=\int_{0}^{1}\left[\frac{1}{2}\left[\alpha|x(s)|^{2}+\beta|x(s)|^{2}\right]+E_{\text {ext }}(x(s)) d s\right]
$$

Where, $\alpha$ is the tension, $\beta$ is the rigidity factor, $E_{\text {ext }}$ is the external energy and $x(s)$ are the snake points. The energy of the image is positive in homogeneous regions and non-zero at the sharp edges. The edge function can be modeled as

$$
g(|\bar{v} u|)=\frac{1}{1+\left|G_{\sigma} * U\right|^{p}}
$$

Where $g$ is a positive decreasing function, $U$ is the image to be segmented, $G_{\sigma}$ is the 2-D Gaussian kernal and ' $P$ ' is an integer (normally chosen to be 2 ). These equations can be solved to obtain the new snake coordinate points $\mathrm{X}$ and $\mathrm{Y}$.

$$
\begin{aligned}
\mathbf{x}^{\langle i+1\rangle} & =\left(\mathbf{A}+\frac{1}{\Delta} \mathrm{I}\right)^{-1}\left(\frac{1}{\Delta} \mathbf{x}^{\langle i\rangle}+f x\left(\mathbf{x}^{\langle i\rangle}, \mathbf{y}^{\langle i\rangle}\right)\right. \\
\mathbf{y} \mathbf{x}^{\langle i+1\rangle} & =\left(\mathbf{A}+\frac{1}{\Delta} \mathrm{I}\right)^{-1}\left(\frac{1}{\Delta} \mathbf{y} \mathbf{x}^{\langle i\rangle}+f y\left(\mathbf{x}^{\langle i\rangle}, \mathbf{y}^{\langle i\rangle}\right)\right.
\end{aligned}
$$

where $A$ is the first order differential of the edge magnitude along $X$-axis and $Y$-axis respectively. These equations can be solved to arrive at the new vector which controls the moment of the snake. 
Madhukumar.S and N.Santhiyakumari; A Novel Segmentation and Contouring Scheme to Assist Accurate Brain Lesion Classification, Journal of Biomedical Engineering and Medical Imaging, Volume 1, No 6, Dec (2014) , pp 1-9

\section{Results and Discussion}

The performance of shape sensitive derivative approach and region growing are compared here. The characterization of the lesion into benign and malignant is done based on the characteristics of the contour and features of the segmented lesion.
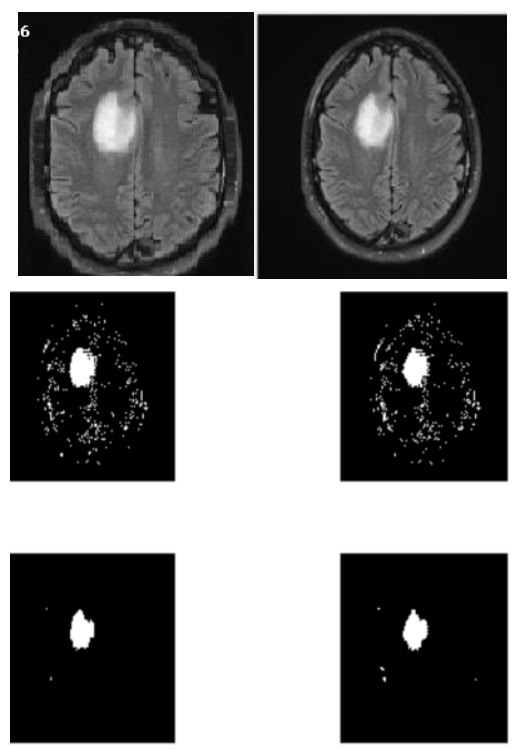

Figure 1: Original and Segmented glioma images

Ten MR image data sets, comprising four true positive glioblastoma multiforme and six true negative, which are benign lesions, of T1 contrast T2weighted and FLAIR sequences has been analysed for Statistical parameters, sensitivity, specificity, positive predictive value and negative predictive value, to evaluate the efficacy of the proposed segmentation method. The geometric features like irregularity of the boundary carries information regarding tumor growth rate prognosis. Such shape based features acting as input to the classifier determine the accuracy of the classification. The accuracy of these shape based features depends on the efficiency of segmentation to preserve the characteristics boundary of ROI. The statistical evaluation indices used to evaluate the features are estimated as sensitivity = number of TP/number of cancer cases; specificity= number of TN/number of non-cancer cases; positive predictive value (PPV) = number of cancer cases/ (number of TP+FP cases); and negative predictive value $(\mathrm{NPV})=$ number of non-cancer cases/ (number of FN+TN). It is expected that the classification efficiency is the mere reflection of the statistical significance of the feature used for classification. It is perspective that the hybrid approach is exhibiting the highest performance index.

Table 1: Statistical Performance Indices

\begin{tabular}{|c|c|c|}
\hline & LDA & $\mathrm{DA}+\mathrm{SVM}$ \\
\hline Sensitivity & $69 \%$ & $90 \%$ \\
\hline Specificity & $77.08 \%$ & $93.3 \%$ \\
\hline Positive Predictive Value & 1.35 & 1.61 \\
\hline Negative Predictive Value & 0.52 & 0.94 \\
\hline \multicolumn{3}{|c|}{ Region Growing Technique } \\
\hline Sensitivity & $62 \%$ & $83 \%$ \\
\hline Specificity & $76 \%$ & $91.3 \%$ \\
\hline Positive Predictive Value & 1.15 & 1.52 \\
\hline Negative Predictive Value & 0.45 & 0.92 \\
\hline
\end{tabular}


The numerical values of the sensitivity, specificity, positive and negative predictive values of LDA and $\mathrm{DA}+$ SVM classifiers for both of the segmentation schemes are shown in table 1 . The original axial plane MR images lesions and the segmented lesions before and after morphological operations are shown in figure 1. From the graphical illustration in figure 2 , it is obvious that the classification DA + SVM overrides LDA

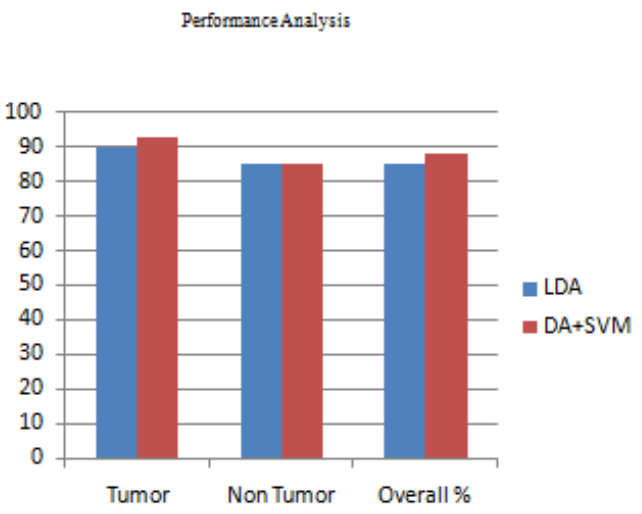

Figure 2: Classification efficiency

\section{Conclusion}

The lesions in the MR images were characterized into benign and malignant ones using shape based features extracted from the segmented lesions. The performance of region growing and sensitive derivative approaches were compared in preserving the geometrical features of the contour. The feature extracted from the segmented lesions were used as input to LDA and DA+SVM classifiers. Shape sensitive derivative approach will preserves the characteristics of the lesion boundary than region growing. The sensitivity, specificity, positive and negative predictive values of shape sensitive derivative segmentation method is more than the region growing. The DA+SVM has better classification ability than LDA.

\section{ACKNOWLEDGEMENT}

The authors would like to thank Dr. Jose Tom (Professor \& Head, Department of Radiotherapy, Government Medical College, Kottayam, India), Dr. Unni.S.Pillai (Department of Oncology, The Jawaharlal Institute of Postgraduate Medical Education \& Research (JIPMER), India), Dr. GowriShanker (Hindlab,Govt. Medical College, Kottayam, India) for their incessant involvement in this endeavor.

\section{REFERENCES}

[1]. W. Gonzalez, Digital Image Processing, 2nd ed. Prentice Hall, Year of Publication, 2008: 378.

[2]. M.LToure, Advanced Algorithm for Brain Segmentation using Fuzzy to Localize Cancer and Epilepsy Region, International Conference on Electronics and Information Engineering (ICEIE) 2010.2. 
Madhukumar.S and N.Santhiyakumari; A Novel Segmentation and Contouring Scheme to Assist Accurate Brain Lesion Classification, Journal of Biomedical Engineering and Medical Imaging, Volume 1, No 6, Dec (2014) , pp 1-9

[3]. OZ.Shi and K.B.Fung, A Comparision of Digital Speckle Filter in proc. IEEE on IGARSS, 1994.4: 2129 - 2133.

[4]. MMancas, BGosselin, Benoîtmacq, Segmentation Using a Region Growing Thresholding Proc. SPIE 5672, Image Processing: Algorithms and Systems IV, 2005:388.

[5]. SanthiyaKumari.N and M.Madheswaran, Analysis of Arthrosclerosis for identification of cerebro-vascular and cardiovascular diseases using active contour segmentation of carotid artery, Proceedings of International symposium on global trends in Bio-medical Informatics, Research, Education and Commercialization, SBRI, India, 2008.

[6]. Djerou, L., Khelil, N., Batouche, M., Image Segmentation by Self-Organised Region Growing, Computer Information Systems and Industrial Management Applications, CISIM '08. 2008:171-176.

[7]. WankaiDeng, Wei Xiao, Chao Pan, J Liu Key, MRI brain tumor segmentation based on improved fuzzy cmeans, Method. Laboratory of Education Ministry for Image Processing and Intelligence Control Institute for Pattern Recognition and Artificial Intelligence SPIE, 2009.7497.

[8]. Auroux, M. Masmoudi, and L. Belaid, Image restoration and classification by topological asymptotic expansion, Variational Formulations in Mechanics: Theory and Applications - CIMNE, Barcelona, Spain 2006.

[9]. Larrabide.I, A. A. Novotny, R. A. Feijo, and E. Taroco, A medical image enhancement algorithm based on topological derivative and anisotropic diffusion, in Proceedings of the XXVI Iberian Latin-American Congress on Computational Methods in Engineering - CILAMCE ,2005.

[10]. Novotny.A, R. Feij'oo, E. Taroco, and C. Padra, Topological sensitivity analysis, Computer Methods in Applied Mechanics and Engineering, 2003. 192: 803-829.

[11]. Kelly H Zou, Statistical Validation of Image Segmentation Quality Based on a Spatial Overlap Index, AcadRadiol. 2004. 11(2): 178-189.

[12]. Ignacio Larrabide, Antonio, Masmoudi, et.al, Topological Derivative as a tool for image processing, Part I, National Laboratory for Scientific Computation, Petr'opolis, Brazil, 2006.

[13]. J Xu, A Janowczyk, S Chandran, A Madabhushi, A high-throughput active contour scheme for segmentation of histopathological imagery, Medical Image Analysis, 2011.15(6): 851-862

[14]. G Xu, E Segawa, S Tsuji, Robust active contours with insensitive parameters, Pattern Recognition, 1994.27(7):879-884 